\title{
Ship particulate pollutants: Characterization in terms of environmental implication
}

\author{
O. Popovicheva, ${ }^{* a}$ E. Kireeva, ${ }^{b}$ N. Shonija, ${ }^{b}$ N. Zubareva, ${ }^{b}$ N. Persiantseva, ${ }^{a}$ V. Tishkova, ${ }^{c}$ B. Demirdjian, ${ }^{c}$ \\ J. Moldanovád and V. Mogilnikov ${ }^{e}$
}

A major aspect of monitoring the atmosphere is the quantification of man-made pollution and their interactions with the environment. Key physico-chemical characteristics of diesel exhaust particulates of sea-going ship emissions are presented with respect to morphology, microstructure, and chemical composition. Heavy fuel oil (HFO)-derived particles exhibit extremely complex chemistry. They demonstrate three distinct morphological structures with different chemical composition, namely soot, char and mineral/ash. The composition analysis investigates the content of environmentally-dangerous pollutants: metals, inorganic/mineral species, and soluble, volatile organic and ionic compounds. It is found that hazardous constituents from HFO combustion, such as transitional and alkali earth metals (V, Ni, Ca, Fe) and their soluble or insoluble chemical forms (sulfides, sulfates, oxides, carbides), are released together with particles into the atmosphere. The water soluble fraction, more than $27 \mathrm{wt} \%$, is dominated by sulfates and calcium cations. They cause the high hygroscopicity of ship exhaust particles and their possible ability to act as cloud nuclei in humid marine environment.

\section{Introduction}

Shipping represents a major contribution of the international transportation sector in respect to environmental and climate impacts, being among the world's highest polluting combustion sources per quality of fuel consumed. ${ }^{1}$ According to global estimates shipping emits $0.4-1.4 \%$ to the total black carbon (BC) burden and increases the aerosol number concentration up to $25 \%{ }^{2}$ Moreover, ships are a highly concentrated source of carbonaceous particulate pollutants which are emitted into clean marine environment and areas of harbors. It was found that up to $90 \%$ of marine aerosols contain BC contaminations even in the remote oceanic atmosphere. ${ }^{3}$ The highly concentrated plumes of

${ }^{a}$ SINP, Moscow State University, 119991 Moscow, Russia

${ }^{b}$ Chemical Department, Moscow State University, 119991 Moscow, Russia ${ }^{c}$ CINaM (Associated with Universite de la Mediterranee (Aix-Marseille II)), UPR CNRS 3118, Campus de Luminy, Marseille, France

${ }^{d} I V L$ Swedish Environmental Research Institute, SE 40014 Göteborg, Sweden

${ }^{e}$ Gromov Flight Research Institute, 140180, Russia ship particulates are emitted most often within such a distance from the coast that they can play important role in coastal air pollution, and into areas of harbors, contributing to their local air quality. ${ }^{4,5}$ Ship particulates are considered as dangerous pollutants with respect to human health. Recent global assessment of $\mathrm{PM}_{2.5}$-related mortalities indicates that ship PM emissions are responsible for $8 \%$ of mortalities on the global scale annually, with most deaths occurring near coastlines in Europe, East Asia, and South Asia. ${ }^{6}$

Currently, PM emissions from shipping are gaining an increasing attention because of possible impacts on clouds and the radiation budget. Global model simulations of the impacts of ocean-going ships demonstrated that the ship emission particulates can significantly increase the cloud droplet number concentration of low marine clouds. Lauer et al. (2007) ${ }^{2}$ estimated this increase as $5-30 \%$, depending on the ship emission inventory and geographic region. This increase may lead to a decrease of the effective radii of cloud droplets and finally to increased cloud optic thickness by up to $10 \%$. Measurements in the Monterey Ship Track Experiment ${ }^{7,8}$ confirmed that ship

\section{Environmental impact}

According to global estimates, shipping contributes significantly to the total black carbon burden and is gaining increasing attention because of possible environmental and climate impacts which are still little defined in comparison with land-based transport. The realistic estimates of the impact of ship emission particulates require their comprehensive characterization, and, first of all, analysis of those properties which are responsible for interactions with the humid environment. This paper provides the general findings concerning the characterization of ship emission residues in terms of environmental impacts. The specific features are presented in respect to morphology, microstructure, elemental composition, inorganic/mineral/metal chemistry, and volatile, water soluble and ionic compounds. The major emphasis is on providing an in-depth analysis of those combustion residue characteristics which are related or responsible for particulate environmental impact, more precisely on the interaction with water vapor. Water uptake measurements demonstrate the ability of ship emission particulates to act as cloud nuclei in humid marine environment. 
emission particulates may act as nuclei for the formation of cloud droplets and thus affect the life cycle and radiative properties of marine stratus clouds at the top of the marine boundary layer. Ship tracks, changes in the cloud reflectance due to the ship emission, are readily detectable in satellite data as elongated structures. Hobbs et al. (2000) ${ }^{8}$ showed that diesel-powered ships burning marine heavy fuel oil (HFO) produce ship tracks and that $12 \%$ of particles in plumes from these ships may serve as cloud condensation nuclei $(\mathrm{CCN})$. Therefore, an appropriate starting point for evaluating the effects of ship emission, and specifically the ship track phenomenon, is to document the nature of PM responsible for interactions of ship-emitted particles with humid environment.

The water content of aerosols and, more fundamentally, affinity of various solutes on the particle surface to water, is one of the important factors (along with size) governing the ability of particles to act as CCN. Directly related to the poor knowledge of the ship particulate composition, major uncertainties exist in understanding the detailed role of surface compounds in interaction with water and in prediction of behavior of the shipemitted particles in the moist atmosphere. To characterize these attributes it is necessary to know the total water soluble fraction on the particle surface, nature of hygroscopic species, and hydrophilic functional groups responsible for particle/water interactions.

Concerning health impacts, a significant environmental hazard of ship PM emissions is associated with particles of submicrometer size, responsible for increased lung cancer and cardiopulmonary mortality. ${ }^{9}$ Aerodynamic diameter is an important inhalation dimension in describing aerosols when considering deposition into the human respiratory system. Toxicological studies have shown that pulmonary injury induced by exposure to HFO combustion residues is associated with their high content of transition metals, especially those in the form of species that are readily soluble in aqueous fluids. ${ }^{10}$ Since this toxicological behavior is believed to be directly related to individual particle characteristics, information on the chemical and physical complexity of particles should lead to improved understanding the appropriate toxicological mechanisms.

Carbonaceous particles formed in different combustion sources demonstrate great variability in their surface properties, morphology and composition. This is because their formation depends on combustion conditions, type of fuel and the engine. The basic feature of all carbon blacks is an elemental carbon matrix formed from microcrystallites of graphite. Features that are variable and that diversify the carbonaceous particles include presence of associated organics (mostly soluble organic fraction) and inorganic impurities. ${ }^{11,12}$ It is assumed that organic compounds are captured by the carbonaceous particles during the cooling phase in the exhaust pipe of a diesel engine. The microstructure and surface chemistry of combustion-generated particulates are highly responsible for their interaction with water and have significant effects on their fate in environment, which is widely unknown. Just recently, some studies of summertime carbonaceous aerosols collected in the arctic marine boundary layer ${ }^{13,14}$ highlighted ship emissions as a potentially important contributor to the morphology and chemistry of particulate matter even in the remote atmosphere.
This work seeks the continuation of ship particulate pollutant studies devoted to investigation of the key physico-chemical characteristics and properties of ship exhaust potentially relating to environmental impacts of sea-going emissions. These findings are based on PM sampled in the hot exhaust of a large diesel engine using HFO. The major emphasis of the methodology elaborated for laboratory studies are placed on providing an indepth analysis of those combustion nanoparticle characteristics which are related to or responsible for particle environmental impact, more precisely on interaction with water vapors. Therefore, our investigations are focused on experimental characterizations of key physico-chemical properties of ship residues highly related to their hygroscopicity. The particle microstructure, elemental composition, chemical forms of impurities, surface chemistry, and water uptake are determined. The composition analyses investigate the content of elemental and organic carbon, metals, inorganic/mineral species, and soluble organic and ionic compounds. Hydrophilic chemical functionalities observed on the particle surface support high water uptake demonstrating the hygroscopicity of ship exhaust particulates.

\section{Sampling and analysis}

Sampling was performed onboard a typical combined Ro-Ro and container ship with the main engine a Kincaid B\&W 6L90 GBE 20,2W. The ship left Liverpool on 13/06/2007 and measurements were made on 14/06/2007 in the area of the Celtic Sea, English Channel and the North Sea as a part of a field campaign organized within the EU project QUANTIFY. The exhaust residues for bulk studies were collected on a metal pipe probe stacked into the exhaust pipeline $c a$. 25 meters behind the engine. The sampling took place during period of $c a .38 \mathrm{~h}$ starting at 20:00 UTC 13/6/2007. The probe was directly inside the pipeline, with exhaust gas temperature $c a .350{ }^{\circ} \mathrm{C}$. Other technical parameters of the ship and data concerning the sampling in dilution line are presented elsewhere. ${ }^{14}$

The ship used low-grade marine residual fuel oil as a fuel, also known as bunker oil. As a residual from crude oil refinement, HFO is a highly polluted fuel. Analysis of the fuel content shows the presence of sulfur, vanadium, iron, sodium, nickel, and calcium at $\sim 2,1.3 \times 10^{-2}, 1.3 \times 10^{-3}, 1.7 \times 10^{-3}, 3.5 \times 10^{-3}$, and $3 \times 10^{-4} \mathrm{wt}^{\%} \%$, respectively. ${ }^{14}$ For comparison, distillate/diesel fuel is a cleaner distillate fuel, it typically contains not more than $0.5 \%$ of sulfur and $0.01 \%$ of ash. The size distribution of particles in the diesel exhaust was probed at the same place where the samples for bulk studies were collected. ${ }^{14}$

The morphological characteristic of major importance for the bulk sampled exhaust residues, the specific surface area, was measured by the $\mathrm{N}_{2}$ thermodesorption method. ${ }^{15}$ The morphology and size of individual ship exhaust particles were determined by Transmission Electron Microscopy (TEM) using the phase-contrast imaging method. Experiments were carried out on a JEOL apparatus: a JEM 2000 FX (200 kV) which has a spatial resolution of $2.8 \AA$. Exhaust particles were deposited on copper microgrids coated with an amorphous carbon-holey film. Microstructure (crystalline phase vs. amorphous state) of carbonaceous particles was observed by electron diffraction technique. Analysis of selected area electron diffraction (SAED) 
patterns allowed the identification of the crystallized form of some chemical compounds.

The elemental composition of individual particles was analyzed via an X-ray energy dispersive spectroscope (EDS, TRACOR series II) coupled to the TEM. Chemical elements with atomic number larger than 5 are detectable by this technique at concentrations exceeding $0.1 \mathrm{wt} \%$. These measurements were accompanied by conventional bulk analyses that provided the average composition that the different particle types have contributed to. Chemical elements with atomic number larger than 11 were determined by X-ray fluorescence spectroscopy (XFS) using a scanning vacuum crystal diffraction spectrometer SPECTROSCAN $(40 \mathrm{kV})$ with the detection limit of $1 \mu \mathrm{g} \mathrm{g}^{-1}$.

Analysis of the elemental carbon (EC), organic carbon (OC) and fly ash content of the ship particulates was performed on filter samples taken at the same place where the samples for bulk studies were collected. Evolved Gas Analyses (EVA) by thermaloptical analyzer ${ }^{16}$ and gravimetric analysis were used. Details are given elsewhere. ${ }^{14}$

Chemical composition of the soluble organic fraction (SOF) in the bulk sample was determined by gas chromatography mass spectrometry (GC/MS). To effectively extract the soluble organic compounds of different polarity, three solvents were chosen, namely methanol, hexane, and water. Hexane is an effective solvent to extract non-polar organic matter while methanol is useful for extraction of slightly water soluble organic compounds with some polar functional groups. Water is used to determine the water soluble compounds. The sample of $26 \mathrm{mg}$ was extracted in $2 \mathrm{ml}$ of solvent and then the extract was filtered. A $1 \mu$ portion of each filtrate was analyzed by an Agilent system consisting of a 6890 gas chromatograph with HP-5MS columns and a 5973 mass spectrometer detector using electron impact ionization. The columns are $30 \mathrm{~m} \times 0.25 \mathrm{~mm}$ i.d. $\times 0.25 \mu \mathrm{m}$ film thickness (HP 5MS, Hewlett Packard). The column temperature was programmed from $70{ }^{\circ} \mathrm{C}$ to $280{ }^{\circ} \mathrm{C}$ at a rate of $20{ }^{\circ} \mathrm{C} \mathrm{min}-1$. The identification of compounds in the SOF was made using the mass spectra library NIST98. Chromatographic peaks with a mass spectral match of $90 \%$ or greater were considered as possible components of the particle extract.

Fractionation of volatile organic compounds in the bulk sample was carried out on a Perkin Elmer 910 gas chromatograph using four different columns and flame ionization and thermion detectors to identify both oxygen and nitrogen containing species. A sample of $2 \mathrm{mg}$ was deposited into the thermodesorber and heated up to $300{ }^{\circ} \mathrm{C}$ with a rate $6{ }^{\circ} \mathrm{C} \mathrm{min}-1$ in nitrogen. The accuracy of measurements was $5-7 \%$. The concentration of inorganic and organic ions was determined by ion chromatography (IC) in an aliquot of the water extract. Anions and cations were detected after sonicating in Dionex ISC3000 and ISC-2000 ion chromatographs, respectively, with an accuracy better than $5 \%$.

The chemical nature of the particles was studied by Fourier Transmission Infrared Spectroscopy (FTIR). FTIR spectra were measured at $4 \mathrm{~cm}^{-1}$ resolution using a Bruker Equinox 55 FTIR spectrometer equipped with a DTGS detector. For this analysis a Ge window was covered by ship exhaust residues. Transmission spectra were recorded by averaging 400 scans in a typical wave number range from 4000 to $400 \mathrm{~cm}^{-1}$.
The microphysical and composition studies completed with water uptake studies allow us to quantitatively examine the role of key particle properties in the interaction with water. Water uptake as a function of relative humidity was measured by a gravimetric method. ${ }^{17}$ To preserve the original surface chemical features, we limited the sample treatment to drying in air at water vapor pressure of $2 \times 10^{-5}$ Torr and did not use the baking treatment. The level of cleaning was controlled by repeatedly weighing the soot sample until the constant weight was attained. The accuracy of water uptake measurements was near $0.05 \mathrm{mmol}$ $\mathrm{g}^{-1}$. In combination with the surface area measurement it provides the basis for quantification of ship exhaust residues.

\section{Results and discussion}

\subsection{Morphology and size distribution}

An important morphological characteristic of any solid object is its surface area. For combustion particles that are the product of incomplete burning of hydrocarbons, commonly called soot, the surface area, S, typically varies from 10 to $100 \mathrm{~m}^{2} \mathrm{~g}^{-1} \cdot{ }^{18,19}$ Smaller surface areas are associated either with organic coverage plugging the soot micropores or with the presence of large primary particles that are not typical soot in nature.

The surface area of the examined ship exhaust residues was found to be low, near $5.4 \mathrm{~m}^{2} \mathrm{~g}^{-1}$, compared to well-studied automobile diesel soot where a range of $\mathrm{S}$ from 20 to $230 \mathrm{~m}^{2} \mathrm{~g}^{-1}$ was reported ${ }^{19,20}$ and to laboratory-made kerosene flame soot with $\mathrm{S} \sim 64 \mathrm{~m}^{2} \mathrm{~g}^{-1} \cdot{ }^{15}$ Consequently a non-soot fraction of the ship exhaust residues having a different (probably, larger) size than typical soot together with the presence of an organic or inorganic fraction covering or filling the micro- and mesoporous particle structures may be proposed. The latter feature has been observed for aircraft engine soot with $\mathrm{S} \sim 6 \mathrm{~m}^{2} \mathrm{~g}^{-1}{ }^{21}$

Such an assumption is consistent with results of measurements of the particulate exhaust size distribution and with direct TEM observations of individual residues. The particle mass size distribution in the ship diesel exhaust was measured and presented in elsewhere. ${ }^{14}$ This mass distribution displays clearly two main aerosol modes: a coarse (median diameter $\mathrm{D}_{\mathrm{p}}>1 \mu \mathrm{m}$ ) and an accumulation $\left(D_{p}<1 \mu \mathrm{m}\right)$ mode, whereas the ultrafine mode $\left(\mathrm{D}_{\mathrm{p}}<0.1 \mu \mathrm{m}\right)$ is negligible. The coarse particles around $7 \mu \mathrm{m}$ constitute a major mode of the ship exhaust residue mass distribution. This distribution is different from typical automobile diesel soot ${ }^{20,22}$ where the mass size distribution is dominated by accumulation mode $\left(\mathrm{D}_{\mathrm{p}}>100 \mathrm{~nm}\right)$. However, exhaust aerosols from medium-speed diesel engines operated with HFO also demonstrate the bimodal mass size distribution with the main mode at $0.1 \mu \mathrm{m}$ and a second mode at about $10 \mu \mathrm{m} .{ }^{23}$ The presence of coarse particles along with fine aerosols is probably associated with HFO combustion and may be explained by the varied nature and composition of ship exhaust particulates. The findings of TEM observations below can confirm this assumption.

\subsection{Microstructure}

In general, ship exhaust residues are found to have heterogeneous microstructure and elemental composition. Three distinct 
morphological structures with quite different chemical identities are identified.

Soot-type particles. Some ship exhaust residues exhibit a secondary structure consisting of a large number of agglomerated roughly spherical primary particles, a common morphology for soot. The electron micrograph in Fig. 1 presents the typical soot particles. The mean size of primary particles was calculated from ninety five TEM images assuming the lognormal distribution. The mean diameter of primary particles was found to be $83 \mathrm{~nm}$ with a standard deviation of 1.2. Agglomerates of soot particles are likely to impact to the accumulation mode of exhaust particles.

The diffraction pattern of the soot particles exhibits the diffused $h k$ (10 and 11) rings of aromatic rings with dots on and between the rings (see Fig. 1a). The ring 002 of graphite microcrystallites is not visible on the diffraction pattern, thus we suppose that the primary soot particles consist of randomly distributed graphene layers and do not possess any intergraphene layer long range order. ${ }^{24}$ Therefore, we should assume an almost disordered carbon structure.

In respect to the soot particle formation, ${ }^{25}$ firstly, hydrocarbon fuel degrades (fuel pyrolysis) into small radicals from which the formation of aromatic rings occurs mainly via addition of acetylene and PAHs in fuel-rich areas. The increase of soot nuclei size is supposed to happen by both coalescence of large aromatic structures and surface growth. With the decrease of the temperature in the flame, the primary particles coagulate into chain- or grape-like aggregates (aciniform) and many of them may be subsequently oxidized. Fuel metal impurities are found to exhibit catalytic activity for soot nuclei formation and lead to the formation of an inorganic ash fraction of the soot particles. Typically, the microstructure of combustion soot particles from different sources is a core-shell structure of the concentrically onion-like oriented graphite planes. ${ }^{24,26-28}$ Multiple spherical nuclei surrounded by several graphitic layers are presented in the inner core.

Comparison of ship exhaust soot with commercial black carbon, ${ }^{26}$ with laboratory-produced kerosene flame soot, ${ }^{29}$ and with diesel engine soot $^{30}$ clearly indicates a strongly different feature of its microstructure due to the presence of the dark cores inside amorphous primary particles (see Fig. 1b). Previously, the finer structure inside the primary particles was observed for aerosols emitted by diesel engines operating with HFO. ${ }^{23}$ The strong contrast seen on the particles suggests that the inner core

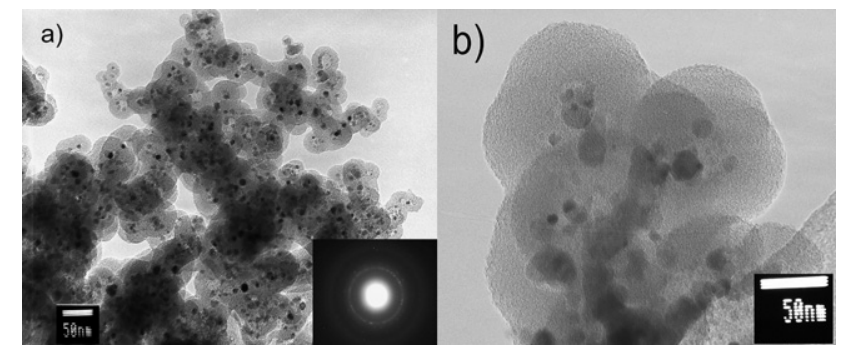

Fig. 1 a) Soot particles in ship exhaust residues polluted by vanadium, nickel, and iron; SAED pattern is inserted. b) Fragment with dark cores inside the amorphous primary particles.

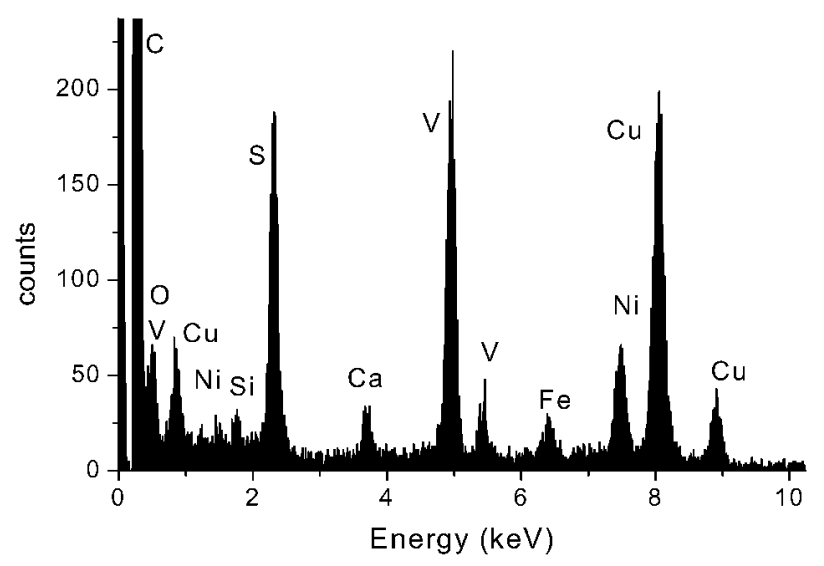

Fig. 2 EDS spectrum of ship soot particles, with carbon, oxygen, vanadium, nickel, calcium, silicon, sulfur, and iron peaks labeled (intense peaks of copper are derived from the TEM copper grid).

consists of more of the heavier elements than the outer core. Moreover, the ship soot particles are found to contain high amounts of vanadium, nickel, and iron, as presented by an EDS spectrum (see Fig. 2). From a comparison based on EDS analysis, ${ }^{31}$ it is inferred that the spectra of soot particles derived from the combustion of fuels with low sulfur content, such as natural gas and gasoline, show only a strong carbon peak and a lower intensity oxygen peak. A discernible sulfur peak in addition to carbon and oxygen peaks are observed in the EDS spectra of soot particles derived from combustion of coal and other fuels containing high amount of sulfur. The presence of metal impurities inside the ship soot particles is likely related to the dark cores on the TEM photos and the dots on the diffraction pattern.

Table 1 presents the typical elemental composition of the ship soot particles measured in thirty areas of the TEM images. Quantitative analysis proves the presence of vanadium at an amount up to $4.5 \%$ which may form crystallized compounds such as $\mathrm{V}_{2} \mathrm{O}_{3}$ found by analysis of the SAED in Fig. 1a. The identification of chemical crystal compounds was performed using a database. ${ }^{32}$ In some particles we have discovered the crystallized structures of $\mathrm{Ni}, \mathrm{Fe}_{2} \mathrm{~S}, \mathrm{Ni}_{2} \mathrm{Fe}$ or $\mathrm{Ni}_{3} \mathrm{Fe}$, and $\mathrm{Ni}_{3} \mathrm{Fe}_{2} \mathrm{O}_{3}$. Therefore, the possibility is that the inner core of the soot particles consists of nucleated metal compounds vaporized from the fuel during the combustion. The following graphitic layers are formed by the condensation of large aromatic structures in a way typical for soot particle formation.

Table 1 Typical elemental content, wt $\%$, of various-type carbonaceous particles in the ship exhaust

\begin{tabular}{llll}
\hline Elements & Soot-type & Char & Char-mineral \\
\hline $\mathrm{C}$ & $90.0 \pm 10.8$ & $79.9 \pm 4.0$ & $13.4 \pm 1.6$ \\
$\mathrm{O}$ & $0.75 \pm 0.08$ & n.d. & $10.8 \pm 0.2$ \\
$\mathrm{~S}$ & $3.5 \pm 0.1$ & $2.3 \pm 0.2$ & $2 \pm 0.1$ \\
$\mathrm{~V}$ & $4.5 \pm 0.2$ & $12.8 \pm 0.2$ & $8.3 \pm 0.3$ \\
$\mathrm{Ni}$ & $1.3 \pm 0.03$ & $4.8 \pm 0.3$ & $1.5 \pm 0.1$ \\
$\mathrm{Si}$ & $0.17 \pm 0.04$ & n.d.* & n.d. \\
$\mathrm{Ca}$ & $0.51 \pm 0.05$ & $0.06 \pm 0.01$ & $63.3 \pm 0.2$ \\
$\mathrm{Fe}$ & $0.35 \pm 0.05$ & $0.1 \pm 0.04$ & n.d. \\
* n.d. not detected. & & & \\
\hline
\end{tabular}


Char particles. HFO combustion particles show some specific differences in morphology comparing to light diesel fuel or kerosene combustion particles. One important feature is the presence of char particles of typically spherical morphology with a size in the range of $0.2-10 \mu \mathrm{m}$. The other difference in morphology is the very prominent agglomerated structure of soot particles composed from the chain of small primary particles, while char particles always appeared as single separate spherules. These specific morphological features are clearly related to the method of particle formation. In contrast to the fuel pyrolysis in the case of soot particles, char particles originate from incomplete combustion of less volatile fractions of HFO; the unburned fuel droplets have undergone oxidation and subsequent carbonization. ${ }^{13,24}$ Also the char particles are composed of microcrystallites of a bigger size that those of soottype particles and often show an anisotropic structure. ${ }^{\mathbf{1 0}}$

A typical char particle found in the ship exhaust residues is shown in Fig. 3. On its surface, some small soot particles are adhered and/or coagulated. The EDS composition of this char particle is presented in Table 1. It is dominated by carbon, but vanadium and sulfur compose as much as $12 \%$ and $2.3 \%$ of mass, respectively. It is worth noting the EDS spectra of char particles in carbonaceous aerosols collected in the marine boundary layer observed by Xie et al., 2007. They are similar to those found in our study.

Moreover, frequently we found some particles characterized by a high content of calcium and vanadium, and by only a minor content of carbon and sulfur. Fig. 4 presents a typical example of this kind of particle, where carbon contributes only $13 \%$ of the mass (see Table 1). Its SAED pattern shows broad rings with numerous dots corresponding to structures of $\mathrm{CaO}, \mathrm{CaSO}_{4}$, and $\mathrm{CaCO}_{3}$. In some particles, the oxide $\mathrm{NiO}$ and sulfides $\mathrm{Ni}_{2} \mathrm{~S}$, and $\mathrm{Ni}_{3} \mathrm{~S}$ have been observed. Due to the high mineral content, we call this category of particle char-mineral.

Mineral/ash particles. Finally, some particles found in the ship exhaust residues contain entirely metal and mineral compounds common for ash and dust. Typically they have a mineral dust structure of elongated prismatic or rhombic crystals of various sizes, up to $7 \mu \mathrm{m}$, as shown in Fig. 5. Analysis of the SAED pattern in Fig. 6a shows the structure of $\mathrm{CaO}$. We may assume polycrystalline lime $\mathrm{CaO}$ particles embedded into

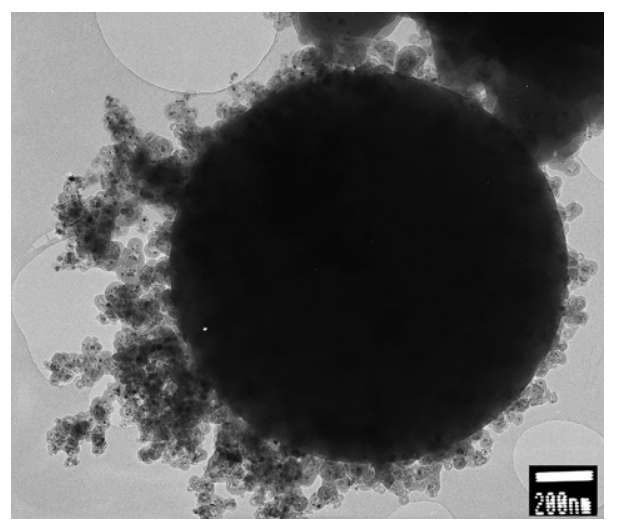

Fig. 3 Char particle surrounded by soot particles observed in ship exhaust residues.

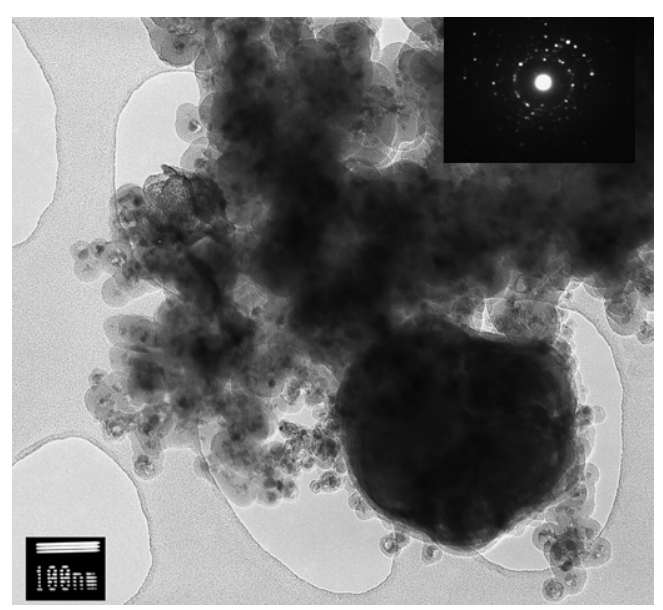

Fig. 4 Char-mineral particles containing heavy elements and its SAED pattern.

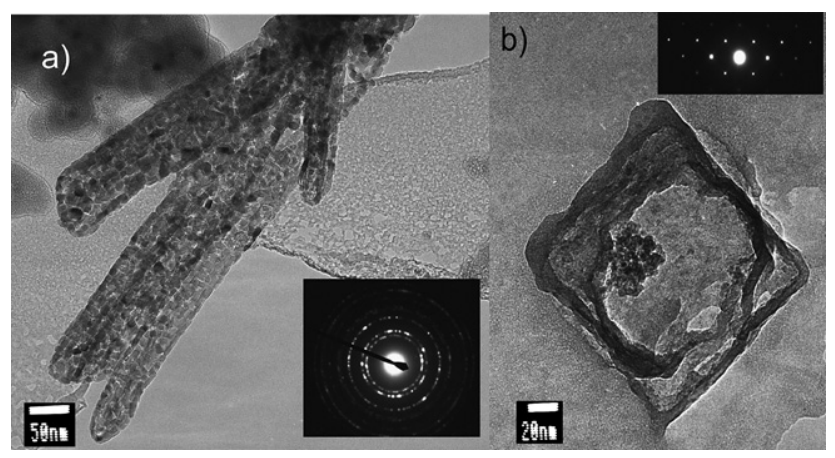

Fig. 5 Mineral/ash particles and its SAED patterns: a) $\mathrm{CaO}$ and b) $\mathrm{Cr}$ particles.

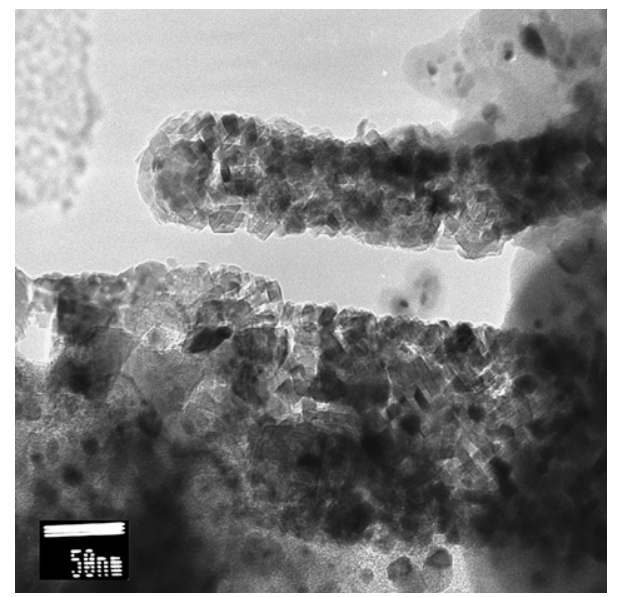

Fig. 6 Complex structure of mineral/ash particles containing $\mathrm{Ca}, \mathrm{Ni}, \mathrm{V}$, and $\mathrm{Fe}$.

a carbonaceous matrix to be randomly oriented nanocrystals. A similar SAED pattern was observed in coal fly ash. ${ }^{33}$

In the aerosols emitted by diesel engines operating on HFO, calcium is commonly observed in the coarse mode of the size distribution, with highest abundance near 5-10 $\mu \mathrm{m} .{ }^{23}$ The 
prominent feature of the element distribution over the different types of particles in the investigated ship residues is the accumulation of a significant amount of calcium in the char-mineral and especially in the mineral/ash particles. Ca produces structures associated with ash or dust particles comprising regular spherical grains, generally $>100 \mathrm{~nm}$, embedded in larger asymmetrical agglomerates resembling minerals.

The presence of well-crystallized $\mathrm{Cr}$ particles is inferred from the analysis of the SAED pattern in Fig. 5b. But more frequently we observe extremely complex polluted agglomerates containing $\mathrm{Ca}, \mathrm{Ni}, \mathrm{V}$, and $\mathrm{Fe}$, with the structure similar to that presented in Fig. 6. Their SAED pattern shows crystallized compounds of $\mathrm{CaV}_{3} \mathrm{O}_{7}, \mathrm{NiFe}_{2} \mathrm{O}_{4}, \mathrm{Ni}\left(\mathrm{CO}_{3}\right)(\mathrm{OH})_{2}, \mathrm{Ni}_{2} \mathrm{Fe}, \mathrm{Ni}_{3} \mathrm{Fe}$, and carbides $(\mathrm{V} ; \mathrm{Fe}) \mathrm{C}$; they have been rarely found in the particulate emission from other transport modes. Their formation may be explained by coalescence of molten grains of minerals during the combustion. Therefore, the presence of these inorganics in a coarse size mode may constitute an important fingerprint useful to identify ship emission sources in the atmosphere.

\subsection{Elemental composition}

Elemental bulk composition inferred from XFS analysis confirms the individual particle composition revealed by the EDS data. The HFO-derived carbonaceous particulates are found to be coated/internally mixed with species containing sulfur, calcium, and transition metals, such as $\mathrm{V}, \mathrm{Ni}, \mathrm{Fe}, \mathrm{Mn}, \mathrm{Cu}, \mathrm{Zn}$, $\mathrm{Cr}$, $\mathrm{Co}$, and $\mathrm{Pb}$. Table 2 presents the bulk content of those elements which were determined quantitatively by the XFS.

Sulfur is often observed in combustion products produced by burning different sulfur-containing fossil fuels in different engines: diesel fuel ${ }^{26,34}$ and aviation kerosene. ${ }^{29}$ This contaminant is likely to be incorporated into the particles during their formation and/or at the last stage of the particles' travel through the exhaust system, when condensation of sulfur-containing gases takes place upon cooling.

Presence of transition metals (e.g., V, Ni) in the ship residues is related to the parental HFO. These pollutants were also observed in ship emitted carbonaceous aerosols ${ }^{13}$ and, in contrast, were not associated with particles collected in diesel truck exhaust, as distillate diesel fuels have only a negligible content of transition metals. ${ }^{24}$ The metal contaminations may influence the catalytic growth of carbon particulates in the combustion area. ${ }^{25}$ This assumption agrees with our observation of dark cores, probably formed by metal oxides inside the soot particles. Moreover, the soot particles appeared to be less polluted by transition metals

Table 2 Elemental composition and ionic species of ship exhaust residues obtained by XFS and IC

\begin{tabular}{llllll}
\hline Element & $\mathrm{wt}^{\%} \%$ & Cation & $\mathrm{wt}^{\circ} \%$ & Anion & $\mathrm{wt} \%$ \\
\hline $\mathrm{V}$ & 1.1 & $\mathrm{Na}^{+}$ & 0.92 & $\mathrm{~F}^{-}$ & 0.18 \\
$\mathrm{Mn}$ & 0.009 & $\mathrm{NH}_{4}^{+}$ & 0.26 & $\mathrm{Cl}^{-}$ & 0.92 \\
$\mathrm{Fe}$ & 0.17 & $\mathrm{~K}^{+}$ & 0.33 & $\mathrm{SO}_{4}{ }^{2-}$ & 16 \\
$\mathrm{Ni}$ & 0.35 & $\mathrm{Mg}^{2+}$ & 0.3 & $\mathrm{NO}_{3}^{-}$ & 0.04 \\
$\mathrm{Zn}$ & 0.024 & $\mathrm{Ca}^{2+}$ & 7.75 & $\mathrm{CH}_{3} \mathrm{COO}^{-}$ & 0.44 \\
$\mathrm{Cr}$ & 0.05 & & & $\mathrm{PO}_{4}^{3-}$ & 0.02 \\
$\mathrm{Co}$ & 0.001 & & & $\mathrm{C}_{2} \mathrm{O}_{4}^{-}$ & 0.13 \\
$\mathrm{~Pb}$ & 0.005 & & & $\mathrm{HCOO}^{-}$ & 0.14 \\
\hline
\end{tabular}

than the char particles. Since the char particles do not undergo an evaporation-condensation process during formation (as soot particles do), inorganic elements from the parent oil fuel (e.g. V, $\mathrm{Ca}, \mathrm{Ni}, \mathrm{S}$ ) are more readily preserved there. The presence of crystalline vanadates, nickel sulfate, sulfides, and oxides in the HOF combustion residues is assumed to serve as a marker of the ship emission. ${ }^{10,33}$

Analysis ${ }^{33}$ has shown that lime $\mathrm{CaO}$ nanocrystals, also found in this study, may be formed from organically bound calcium in the HFO. Similar nanocrystals were also found in coal fly ash, while a negligible amount of $\mathrm{Ca}, c a .0 .03 \%$, was found in the particulate residues from a medium duty diesel truck. ${ }^{34}$ The diesel fuel oil lacks any detectable calcium impurities. Moreover, a significant fraction of the background atmospheric particulate matter consists of mineral dust with abundant calcium-rich particles. Limestone particles are generated through its application in manufacturing and building, and may pollute the ocean air mass near harbours and coasts. ${ }^{35}$ The elongated prismatic crystals found in the marine boundary layer ${ }^{3,13}$ contain $\mathrm{Ca}$ associated with calcite $\left(\mathrm{CaCO}_{3}\right)$ and gypsum $\left(\mathrm{CaSO}_{4} \cdot 2 \mathrm{H}_{2} \mathrm{O}\right)$. Their similarity with particles observed in this study allows the assumption that some calcium-rich minerals may enter a ship engine with polluted air and form the internal mixture of presumably polymorphs of anhydrate and calcium carbonate. Finally, the main additive component of the lubricating oil in large ship engines, $\mathrm{Ca}$, that makes up to $1 \mathrm{wt} \%,{ }^{23}$ can be volatilized in the engine cylinder during the combustion and then can be found in the particles together with other fuel heavy elements.

\subsection{Organic content}

Analysis of the ship particle composition with respect to the content of $\mathrm{EC}, \mathrm{OC}$, and ash shows $\mathrm{OC} / \mathrm{EC} \approx 2.8$ and $\mathrm{OC} / \mathrm{ash} \approx$ 1. ${ }^{14}$ Such a high content of organic carbon and ash in the ship residues strongly distinguish these combustion products from commercial carbon blacks (where EC typically composes near $95 \mathrm{wt} \%$ ) and from automobile diesel soot (where EC composes $75-80 \mathrm{wt} \%) .{ }^{26}$ The large amount of organic compounds requires their fractionation into classes in order to further characterize organic matter with respect to its hygroscopicity.

A comprehensive evaluation of organics present in diesel soot,${ }^{34}$ motor vehicle soot, ${ }^{36}$ and aircraft engine combustor soot ${ }^{37}$ pointed out the different classes of $\mathrm{C}_{1}$ through $\mathrm{C}_{30}$ organic compounds. Motor vehicles are regarded as one of the more important sources of soot-associated polyaromatic hydrocarbons (PAH), contributing the most nanoparticle mass. ${ }^{11,36}$ Lightduty diesel soot exhibits heavy alkanes constituting from 30 to $60 \%$ of the organic mass as a function of engine operating conditions. ${ }^{38} \mathrm{GC} / \mathrm{MS}$ analysis facilitates the differentiation of organics into fractions. The particulate-phase organic compounds were quantified in medium duty diesel exhaust, such as normal and branched alkanes, saturated cycloalkanes, aromatic hydrocarbons, n-alkanoic, alkanedioic, and aromatic acids. ${ }^{34}$ Motor vehicle organic soot matter was identified to consist of alkanes, organic acids, PAHs, and oxygenated PAHs. ${ }^{36}$

Our GC/MS analysis of organic compounds in ship exhaust residues allows their separation into the following main classes: alkanes, alkenes, polyaromatics, esters, anhydrides, azoles and amines. Fractionation of particle-phase organics extracted by 
Table 3 Particle-phase organic compounds of ship exhaust residues identified by GC/MS

\begin{tabular}{ll}
\hline Class & compounds \\
\hline Alkanes & $\begin{array}{l}\text { Dibromotetrapentacontane, hexadecane } \\
\text { Heptadecane } \\
\text { 1-Nonadecene } \\
\text { Tetracosapentaene, hexamethyl- } \\
\text { Alkenes }\end{array}$ \\
Benzo[a]pyrene, Perylene \\
Polyaromatics & Benzo[e]acephenanthrylene \\
Esters & Hexadecanoic acid, methyl ester \\
& 1,2-Benzenedicarboxylic acid diisooctyl ester \\
& Decanedioic acid, dibutyl ester \\
& Dibutyl phthalate \\
& Phthalic anhydride \\
Anhydrides & Benzothiazole \\
Azoles & Benzenamine, $N$-phenyl \\
Amines &
\end{tabular}

methanol, hexane, and water is presented in Table 3. Some oxygen-containing compounds like esters, anhydrides, and azoles are found. These compounds are more polar compounds than alkanes, alkenes, and PAHs. However, their large hydrophobic aliphatic or aromatic parts do not allow the classifying of these compounds as hydrophilic.

Analysis of compounds that are volatile at $300{ }^{\circ} \mathrm{C}$ shows only non-oxygen containing $\mathrm{C}_{6}-\mathrm{C}_{9}$ aromatic hydrocarbons, like benzene, toluol, isomers of xylol (dimethylbenzene) and cumene (phenyl propane), and aliphatic components of HFO such as heptadecane and hexadecane, and benzopyrene. A very small fraction, $0.3 \mathrm{wt} \%$, of volatile organics was measured. A large fraction of non-extractable compounds (resin) remained unresolved; it is nonvolatile at temperatures $<300{ }^{\circ} \mathrm{C}$.

\subsection{Water soluble species}

For hygroscopicity studies, the most interesting issue is the identification of oxygen-containing and water soluble compounds on the particle surface. These compounds strongly affect the interaction of ship exhaust particulates with water. Solvent-extractable and volatile compounds identified by $\mathrm{GC} /$ MS and gas chromatography are not hydrophilic and are slightly water soluble species. Probably, the most hygroscopic compounds of the ship residues are those providing ionic species when immersed into water. They may be low molecular weight organic acids, inorganic acids, and salts.

The data obtained by IC are summarized in Table 2 . The total mass of anionic and cationic species makes up as much as $27 \mathrm{wt} \%$. Ionic species in ship residues are dominated by sulfate anions, $16 \mathrm{wt} \%$, and calcium cations, $7.75 \%$. The high content of $\mathrm{Ca}^{2+}$ leads us to the conclusion that a large fraction of sulfates is associated to anhydrite or gypsum $\left(\mathrm{CaSO}_{4}\right.$ or $\left.\mathrm{CaSO}_{4} \cdot 2 \mathrm{H}_{2} \mathrm{O}\right)$. Some sulfate anions may also be associated to $\mathrm{Na}_{2} \mathrm{SO}_{4}, \mathrm{~K}_{2} \mathrm{SO}_{4}$, $\left(\mathrm{NH}_{4}\right)_{2} \mathrm{SO}_{4}$ and $\mathrm{MgSO}_{4}$ sulfate salts that make up in total 4.42 $\mathrm{wt} \%$, as the corresponding cations are found in the water extract. Some amount of sulfates may be associated to sulfuric acid accumulating on the particulate surface due to condensation in the exhaust or to binary heterogeneous nucleation of $\mathrm{H}_{2} \mathrm{SO}_{4}$ / $\mathrm{H}_{2} \mathrm{O},{ }^{39}$ and due to coagulation of primary combustion particles with nucleated sulfate aerosols.
The ionic composition of the residue water extract reveals small impurities of organic ions such as formate $\mathrm{HCOO}^{-}$, acetate $\mathrm{CH}_{3} \mathrm{COO}^{-}$, and oxalate $\mathrm{C}_{2} \mathrm{O}_{4}{ }^{-}$, in total $0.71 \mathrm{wt} \%$. Electrolytic inorganic substances such as nitrate $\mathrm{NO}_{3}{ }^{-}$, chloride $\mathrm{Cl}^{-}$, fluoride $\mathrm{F}^{-}$, and phosphate $\mathrm{PO}_{4}{ }^{3-}$, in total $1.16 \mathrm{wt} \%$, indicate the presence of inorganic salts which are highly-soluble in water. For comparison, the amount of sulfate and ammonium ions found in the particle emission from medium duty diesel truck is just 1.0 and $0.73 \mathrm{wt} \%$, respectively. ${ }^{34}$

Electron diffraction analysis, described above, clearly confirms the presence of water-soluble and hygroscopic compounds like anhydrite $\mathrm{CaSO}_{4}$ and lime $\mathrm{CaO}$. The most hygroscopic among the detected species is lime, the reaction of which with water, $\mathrm{CaO}+2 \mathrm{H}_{2} \mathrm{O}=\mathrm{Ca}(\mathrm{OH})_{2}$, is strongly exothermic. Calcite $\mathrm{CaCO}_{3}$, also identified by electron diffraction, is slightly soluble and relatively hydrophobic. Other identified metal sulfides, oxides, and carbides are insoluble in their chemical forms.

With respect to inhalability of the particulate matter, the submicron aerosol particles present a more significant negative health effect. Hazardous constituents from the HFO such as transitional and alkali earth metals $(\mathrm{V}, \mathrm{Ni}, \mathrm{Fe}, \mathrm{Mn}, \mathrm{Cu}, \mathrm{Zn}, \mathrm{Cr}$, $\mathrm{Co}, \mathrm{Pb}$, and $\mathrm{Ca}$ ), and their soluble and insoluble chemical forms (sulfides, oxides, carbides) are released into the atmosphere together with the ship emitted micrometer particles, and these soluble forms are generally regarded as potentially increasing the PM adverse effects.

\subsection{Surface chemistry}

The surface of black carbons can have acidic or basic properties; the acidic ones are caused by the presence of H-containing carboxyl and hydroxyl groups. ${ }^{40}$ The oxygen-containing functionalities such as carboxyl, hydroxyl, and carbonyl in anhydrides, ethers, lactones, and quinones may create the hydrogen bonds with water molecules. The different strengths of these hydrogen bonds impacts the extent of soot hygroscopicity. ${ }^{\mathbf{4 1 , 4 2}}$ The acidic carboxyl groups are strongest with respect to the hydrogen bond, near 15-20 $\mathrm{kJ} \mathrm{mol}^{-1}$. $^{4344}$ The hydroxyl group in carboxyl is stronger than in phenols with respect to its protondonor ability and polarity of the $\mathrm{O}-\mathrm{H}$ bond. The carbonyl group is stronger than the oxygen-containing group in esters and ethers. ${ }^{42,45}$ Within polar functional groups, ionic species are the most effective with respect to the highest ability to chemosorb the water molecules. The energy of the hydrogen bond for sulfate anions is estimated to be around $33.4 \mathrm{~kJ} \mathrm{~mol}^{-1}$. 46

Fig. 7 shows the FTIR spectrum of the ship exhaust particles. One of the most prominent features is the peaks at 590 and $670 \mathrm{~cm}^{-1}$, a strong evidence of sulfate ions. ${ }^{47}$ This finding is in good agreement with the large amount of $\mathrm{SO}_{4}{ }^{2-}$ ions found in the water extracted from these residues. The EDS observations of sulfur in/on various particles allow the assignment of the bands found around 1380 and $1080 \mathrm{~cm}^{-1}$ to the $\mathrm{SO}_{2}$ group vibrations. ${ }^{48}$ The other less prominent bands at $610 \mathrm{~cm}^{-1}$ and $980 \mathrm{~cm}^{-1}$ can be assigned to $\mathrm{C}-\mathrm{S}$ and $\mathrm{V}=\mathrm{O}$ vibrations in sulfides and vanadium oxide, respectively. ${ }^{48,49}$ Features of organic sulfones and sulfates can be distinguished near 1145 and $1380 \mathrm{~cm}^{-1}$. The bands near $1560 \mathrm{~cm}^{-1}$ and $1680 \mathrm{~cm}^{-1}$ indicate $\mathrm{C}=\mathrm{O}$ functionalities, ${ }^{46}$ probably in quinines, esters, and anhydrides as these classes of organics were found by GC/MS. Moreover, the bands near 


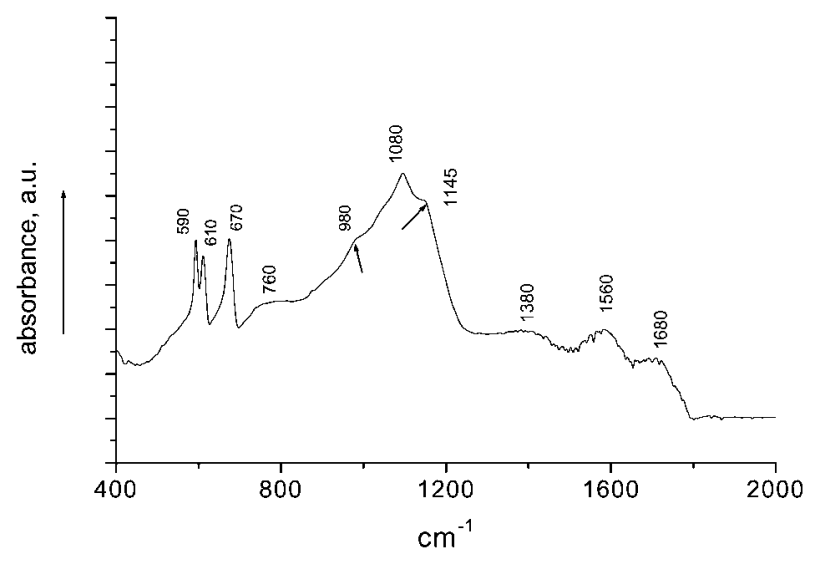

Fig. 7 FTIR spectra of ship exhaust residues.

$1380 \mathrm{~cm}^{-1}$ and $1560 \mathrm{~cm}^{-1}$ may be assigned to $-\mathrm{N}=\mathrm{N}-$ aromatic and aliphatic stretching vibrations, respectively, in azolic compounds. ${ }^{50}$ A C-N-C asymmetric stretching vibration in amines may impact the small peak at $1145 \mathrm{~cm}^{-1} .^{50}$ Finally, $\mathrm{C}-\mathrm{H}$ out-of-plane deformation of condensed aromatic compounds may be responsible for a band near $760 \mathrm{~cm}^{-1} \cdot{ }^{51}$ Absence of other features of aromatic compounds in the spectrum should not mislead to the conclusion that PAHs are not significant on ship exhaust particles because many PAHs were identified by the GC/ MS. The FTIR bands of the different surface compounds strongly overlapped, leading to a broad combination with bad resolution.

Oxygen and sulfur-containing functional groups available for hydrogen bond formation allow the quantification of the nature of primary adsorption centers for water, determining the extent of the particle hydrophilicity. The stronger hydrogen bonding corresponds to more hydrophilic functional groups, the hydrophobic groups may form fairly weak hydrogen bonds. From FTIR analysis we may propose the hydrophilicity of ship exhaust residues because of the presence of many hydrophilic groups on their surface including sulfur functionalities, also in ionic form.

\subsection{Water uptake: hygroscopicity}

Characterization of ship emission residues allows quantitative clarifying of the role of key particulate properties in the interaction with water. The low surface area indicates a significant impact of non-soot particles (having a size and structure different from agglomerated soot primary particles) and presence of the thick coverage of organic/inorganic surface compounds filling micro- and mesopores. The high water soluble fraction (more than $27 \mathrm{wt} \%$ ) indicates the significant hygroscopicity of ship residues. Finally, the presence of hydrophilic oxygen- and sulfurcontaining functionalities with ionic groups should lead to high water uptake.

Fig. 8 shows the amount of absorbed water on ship residues as a function of the relative humidity $(\mathrm{RH})$. To build the absolute adsorption isotherm for comparative analyses we recalculated the amount of adsorbed water measured in mmole per gram into the amount of statistic monolayers (ML) using the measured surface area $S\left(\mathrm{~m}^{2} \mathrm{~g}^{-1}\right)$ of a given sample. One statistic monolayer was estimated to be equal to $\sim 10$ water molecules per $\mathrm{nm}^{2}$

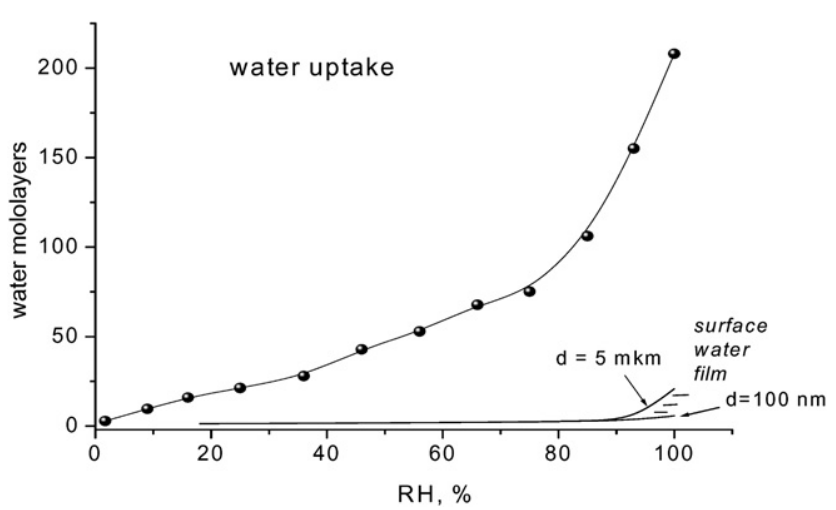

Fig. 8 Isotherm of water uptake by ship exhaust residues. The area of existence for water surface film is indicated, with low and high boundaries for $100 \mathrm{~nm}$ and $5 \mu \mathrm{m}$ diameter particles, respectively.

assuming a cross-sectional area of a water molecule of $0.105 \mathrm{~nm}^{2}$. The formation of a water film extended over the surface was suggested as a quantification measure which separates hygroscopic from non-hygroscopic soot in the concept of quantification of water uptake by combustion particles. ${ }^{21}$ This is why for comparison in Fig. 8 we plot the isotherms for the surface water film on the particles of $100 \mathrm{~nm}$ and $5 \mu \mathrm{m}$ diameters that roughly simulate the mean diameters of the particles in accumulation and coarse modes. One can see that ship exhaust residues demonstrate high water uptake, much higher than is needed to cover the particle surface by a water film. The high water affinity of the surface of ship residues is demonstrated by significant uptake (more than $1 \mathrm{ML}$ ) occurring already at very low RH. If the particle absorption exceeds a few monolayers and the coverage matter consists of water-soluble materials, the water uptake should be treated in terms of bulk dissolution. ${ }^{21}$ Significant uptake (up to $200 \mathrm{ML}$ ) and the presence of many hydrophilic functional groups on the surface of ship residues do not leave any doubt that the dominant mechanism of water uptake is water dissolution into the soluble coverage and formation of the thick solution film surrounding the particles. The role of sulfates and calcium-containing hygroscopic compounds providing volume sites for bulk water uptake may be dominating. Thus, ship exhaust residues may contribute, with highly hygroscopic aerosols, to the atmosphere in respect to the concept of quantification.

\section{Conclusions}

The important finding of this study is that ship exhaust particles produced by burning HFO in a large diesel marine engine are expended in the range up to $10 \mu \mathrm{m}$ and exhibit extremely complex chemistry. Ship particulates have three distinct morphological structures with different chemical composition. Soot aggregates highly polluted by vanadium, nickel, calcium, and sulfur dominate in the submicrometer fraction. The heavy elements present in the fuel form the core of the soot particles. Spherical shaped char particles are abundant in the micrometer size region, whereas irregular shaped mineral/ash particles with a prominent calcium and vanadium content have a size up to $10 \mu \mathrm{m}$. Hazardous constituents from the HFO, such as transitional and 
alkali earth metals (V, Ni, Fe, Mn, Cu, Zn, Cr, Co, Pb, and $\mathrm{Ca}$ ), and their soluble and insoluble chemical forms, are released into the atmosphere together with micrometer particles. Soluble forms are generally regarded as having potentially more adverse health effects than those tied up as insoluble. These inorganic species pollutants may constitute an important fingerprint that could be used to identify the ship emission source of particulates in the atmosphere.

A lot of organics and ash in the ship exhaust residues strongly distinguish these combustion products from commercial carbon blacks and automobile diesel soot. Extractable organic compounds are separated into a few main classes: alkanes, alkenes, polyaromatics, esters, anhydrides, azoles, and amines; all are nonhydrophilic and slightly water soluble. The main constituents of ship residues are water soluble electrolytic inorganic and organic ionic species: they are dominated by sulfate anions and calcium cations. Probably, the most hygroscopic compounds of the ship residues are those providing ionic species when particles are immersed into water. They may be light organic (formic, acetic, oxalic) and inorganic (sulfuric) acids, and sulfate salts like $\mathrm{CaSO}_{4}, \mathrm{Na}_{2} \mathrm{SO}_{4}, \mathrm{~K}_{2} \mathrm{SO}_{4},\left(\mathrm{NH}_{4}\right)_{2} \mathrm{SO}_{4}$, and $\mathrm{MgSO}_{4}$. These species, together with lime $\mathrm{CaO}$, may constitute important ship-emitted aerosol characteristics responsible for water uptake in the humid atmosphere.

Measurements of water uptake by ship exhaust particles demonstrate that it is much higher than is needed to cover the surface with a water film. Significant uptake in correlation with the presence of hygroscopic compounds supports the dominant mechanism of ship residues water uptake as the water dissolution into the soluble coverage, and formation of a thick solution surrounding the particles. Therefore, heavy-fuel-derived particulates may be discovered in the atmosphere as hygroscopic and $\mathrm{CCN}$ active. Knowledge of the chemical composition of the ship particulates and their hygroscopicity can contribute to formulation of improved emission control strategies mitigating negative health effects and climate change.

\section{Acknowledgements}

This research is funded by EU FP6 Project QUANTIFY-TTC 003893, the grant of President of Russian Federation SS133.2008.02, ISTC Project 3097, and CRDF-RFBR Project 2949/09-05-92506-a. T.V.V. thanks to Dr. D.Ferry (CINaM/ CNRS) for help with FTIR measurements.

\section{References}

1 J. J. Corbett and P. Fischbeck, Emissions from ships, Science, 1997, 278, 823 .

2 A. Lauer, V. Eyring, J. Hendricks, P. Jockel and U. Lohmann, Global model simulations of the impact of ocean-going ships on aerosols, clouds, and the radiation budget, Atmos. Chem. Phys., 2007, 7, 5061.

3 P. Buseck and M. Posfai, Airborn minerals and related aerosol particles: effects on climate and the environment, Proc. Natl. Acad. Sci. U. S. A., 1999, 96, 3372.

4 Ø. Endresen, E. Sørgård, J. K. Sundet, S. B. Dalsøren, I. S. A. Isaksen, T. F. Berglen and G. Gravir, Emission from international sea transportation and environmental impact, J. Geophys. Res., 2003, 108, 4560, DOI: 10.1029/2002JD002898.

5 V. Eyring, H. W. Kohler, J. van Aardenne and A. Lauer, Emissions from international shipping: I. The last 50 years, J. Geophys. Res., 2005, 110, D17305, DOI: 10.1029/2004JD005619.
6 J. J. Corbett, J. J. Winebrake, E. H. Green, P. Kasibhatla, V. Eyring and A. Lauer, Mortality from ship emissions. A Global Assessment, Environ. Sci. Technol., 2007, 41(24), 8512, DOI: 10.1021/es071686z.

7 P. Durkee, K. Noone and K. Bluth, The Monterey area ship track experiment, J. Atmos. Sci., 2000, 57, 2523.

8 P. Hobbs, T. Garrett, R. Ferek, S. R. Strader, D. A. Hegg, G. M. Frick, W. A. Hoppel, R. F. Gasparovic, L. M. Russell, D. W. Johnson, P. A. Durkee, K. E. Nielsen, C. O'Dowd and G. Innis, Emissions from ships with respect to their effects in clouds, J. Atmos. Sci., 2000, 57, 2570.

9 B. Balbus et al., Meeting report: hazard assessment for nanoparticles - report from an interdisciplinary workshop, Environ. Health Perspect., 2007, 115, 1654.

10 Y. Chen, N. Shah, F. Huggins and G. Huffman, Investigation of the microcharacteristics of PM 2.5 in residual oil, ash by analytic transmission electron microscopy, Environ. Sci. Technol., 2004, 38, 6553.

11 M. Fernandes, J. Skjemstad, B. Johnson, J. Wells and P. Brooks, Characterization of carbonaceous combustion residues. I. Morphological, elemental and spectroscopic features, Chemosphere, 2003, 51, 785 .

12 M. Fernandes and P. Brook, Characterization of carbonaceous combustion residuals: II. Nonpolar organic compounds, Chemosphere, 2003, 53, 447.

13 Z. Xie, J. D. Blum, S. Utsunomiya, R. C. Ewing, X. Wang and L. Sun, Summertime carbonaceous aerosols collected in the marine boundary layer of the Arctic Ocean, J. Geophys. Res., 2007, 112, D02306, DOI: 10.1029/2006JD007247.

14 J. Moldanová, E. Fridell, O. Popovicheva, B. Demirdjian, V. Tishkova, A. Faccinetto and C. Focsa, Characterization of particulate and gaseous emissions from a large ship diesel engine, Atmos. Environ., 2009, 43, 2632-2641, DOI: 10.1016/ j.atmosenv.2009.02.008.

15 O. B. Popovicheva, N. M. Persiantseva, B. V. Kuznetsov, T. A. Rakhmanova, N. K. Shonija, J. Suzanne and D. Ferry, Microstructure and water adsorbability of aircraft combustor soots and kerosene flame soots: Toward an aircraft-generated soot laboratory surrogate, J. Phys. Chem. A, 2003, 107, 10046.

16 M. E. Birch and R. A. Cary, Elemental carbon-based method for monitoring occupational exposures to particulate diesel exhaust, Aerosol Sci. Technol., 1996, 25, 221.

17 D. Kantro, S. Brunauer, L. E. Copeland, in The Solid-Gas Interface, Marcel Dekker, Inc., New York, 1967, vol. 1, (ch. XII).

18 S. J. Gregg and K. S. W. Sing, in Adsorption, surface area and porosity. Academic Press: New York, 2nd ed, 1982.

19 B. R. Stanmore, J. Brilhac and P. Gilot, The oxidation of soot: a review of experiments. Mechanisms and models, Carbon, 2001, 39, 2247.

20 A. Smekens, R. H. M. Godoi, P. Berghmans and R. van Grieken, Characterization of soot emitted by domestic heating, aircraft, and cars using diesel or biodiesel, J. Atmos. Chem., 2005, 52, 45.

21 O. B. Popovicheva, N. M. Persiantseva, V. Tishkova, N. K. Shonija and N. A. Zubareva, Quantification of water uptake by soot particles, Environ. Res. Lett., 2008, 3, 025009, DOI: 10.1088/17489326/3/2/025009.

22 H. Burtscher, S. Kunzel and S. Huglin, Characterization of particles in combustion engine exhaust, J. Aerosol Sci., 1998, 29, 389.

23 J. Lyyranen, J. Jokiniemi, E. Kauppinen and J. Joutsensaari, Aerosol characterization in medium-speed diesel engine operating with heavy fuel oils, J. Aerosol Sci., 1999, 30, 771.

24 Y. Chen, N. Shah, A. Braun, F. Huggins and G. Huffman, Electron microscopy investigation of carbonaceous particulate matter generated by combustion of fossil fuels, Energy Fuels, 2005, 19, 1644.

25 H. Bockhorn, Soot formation in combustion: Mechanisms and models. Springer, Berlin Heidelberg New York, 1994.

26 A. D. Y. Clague, J. B. Donnet, T. K. Wang and J. C. Peng, A comparison of diesel engine soot with carbon black, Carbon, 1999, 37, 1553 .

27 O. B. Popovicheva, N. M. Persiantseva, M. E. Trukhin, G. B. Rulev, N. K. Shonija, Y. Y. Buriko, A. M. Starik, B. Demirdjian, D. Ferry and J. Suzanne, Experimental characterization of aircraft combustor soot: microstructure, surface area, porosity and water adsorption, Phys. Chem. Chem. Phys., 2000, 2, 4421.

28 J.-O. Muller, D. S. Su, R. E. Jentoft, U. Wild and R. Schlogl, Diesel engine exhaust emission: oxidative behaviour and microstructure of black smoke soot particulate, Environ. Sci. Technol., 2006, 40, 1231. 
29 B. Demirdjian, D. Ferry, J. Suzanne, O. B. Popovicheva, N. M. Persiantseva and N. K. Shonija, Heterogeneities in the microstructure and composition of aircraft engine combustor soot: Impact on the water uptake, J. Atmos. Chem., 2007, 56, 83.

30 J.-O. Muller, D. S. Su, R. E. Jentoft, J. Krohnert, F. C. Jentoft and R. Schlogl, Morphology-controlled reactivity of carbonaceous materials towards oxidation, Catal. Today, 2005, 102-103, 259.

31 Y. Chen, N. Shaha, F. E. Huggins and G. P. Huffman, Microanalysis of ambient particles from Lexington, KY, by electron microscopy, Atmos. Environ., 2006, 40, 651.

32 Mineralogy Database, www.webmineral.com, last accessed October 2008.

33 Y. Chen, N. Shah, F. Huggings and G. Huffman, Transmission electron microscopy investigation of unltrafine coal fly ash particles, Environ. Sci. Technol., 2005, 39, 1144.

34 J. J. Schauer, M. J. Kleeman, G. Cass and B. Simoneit, Measurements of emissions from air pollution sources.2. $\mathrm{C}_{1}$ through $\mathrm{C}_{30}$ organic compounds from medium duty diesel trucks, Environ. Sci. Technol., 1999, 33, 1578

35 R. K. Xie, N. M. Seip, J. R. Leinum, T. Winje and J. S. Xiao, Chemical characterization of individual particles $\left(\mathrm{PM}_{10}\right)$ from ambient air in Guiyang City, China, Sci. Total Environ., 2005, 343, 261.

36 M. Fraser, G. Cass and B. Simoneit, Particulate organic compounds emitted from motor vehicle exhaust and in the urban atmosphere, Atmos. Environ., 1999, 33, 2715.

37 O. B. Popovicheva, E. D. Kireeva, A. V. Kamaev, T. D. Khokhlova, N. K. Shonija, E. V. Vlasenko, Proceeding of Nonequilibrium Processes: Plasma, Combustion. Atmospheric Phenomena Symposium 2007, Roy, G. O., Frolov, S. M., Starik, A. M., Eds.; Moscow, Torus Press, 2007, 109.

38 S. Brandenberger, M. Mohr, K. Grob and H. P. Neukom, Contribution of unburned lubricating oil and diesel fuel to particulate emission from passenger cars, Atmos. Environ., 2005, 39, 6985.

39 H. Tobias, D. Beving, P. Ziemann, H. Sakurai, M. Zuk, P. McMurry, W. Zarling and D. Kittelson, Chemical analysis of diesel engine nanoparticles using a nano-DMA/thermal desorption particle beam mass spectrometer, Environ. Sci. Technol., 2001, 35, 2233.

40 H. P. Boehm, Surface oxides on carbon and their analysis: a critical assessment, Carbon, 2002, 40, 145.

41 G. C. Pimentel, A. L. McClellan, The Hydrogen Bond. in A Series of Chemistry Books, 1960 W.H. Freeman and company, San Francisco and London., 475.

42 G. Curtoys, V. Ya. Davydov, A. V. Kiselev and B. V. Kuznetsov, Hydrogen Bonding in Adsorption on Silica, J. Colloid Interface Sci., 1974, 48(1), 58.

43 Yu. I. Tarasevich, E. V. Aksenenko, S. V. Bondarenko and A. I. Zukova, Complex investigations of cluster adsorption of water molecules on hydrophilic centers of graphite and graphitized thermal carbon blacks, Theor. Exp. Chem., 2007, 43, 197.

44 F. Moulin, P. N. M. Hoang, S. Picaud, L. B. Partay and P. Jedlovszky, A grand canonical Monter Carlo simulation of the aggregation of water molecules on chemical modified soot particles, Computing Lett., 2008, 4, 105.

45 E. J. Kokunova, S. N. Lanin, J. S. Nikitin and H. K. Shonija, Gas chromatography studies of temperature impact on residence time and heat of adsorption of oxygen-containing compounds on silica, J. Phys. Chem., 1993, 67(8), 1680.

46 R. M. Badger and S. H. Bauer, Spectroscopic studies of the hydrogen bond. II. The shift of the $\mathrm{O}-\mathrm{H}$ vibration frequency in the formation of the hydrogen bond, J. Chem. Phys., 1937, 5, 839.

47 G. Socrates, Infrared and Raman characteristic group frequencies, Tables and Charts, John Wiley and Sons., 2004.

48 B. Stuart, Infrared Spectroscopy: Fundamentals and Applications, John Wiley \& Sons, Ltd., 2004.

49 Z. Liu, C. Zhao, A. Xu, L. Duan and X. Zhu, Diesel soot oxidation over supported vanadium oxide and $\mathrm{K}$-promoted vanadium oxide catalysts, Appl. Catal., B, 2005, 61, 36.

50 P. Patnaik and J. A. Dean, Dean's Analytical Chemistry Handbook, 2004, McGraw-Hill.

51 J. A. Jassim, H. P. Lu, A. R. Chughtai and D. M. Smith, The origin of infrared absorption bands of soot in the region 750-900 $\mathrm{cm}^{-1}$, Appl. Spectrosc., 1986, 40(1), 113. 